\title{
Benchmarking the Scientific and Educational Impact of the Annals of Surgical Oncology
}

\author{
Charles M. Balch, MD, FACS ${ }^{1,2}$, V. Suzanne Klimberg, MD $^{3}$, Kelly M. McMasters, MD, $\mathrm{PhD}^{4}$, \\ Timothy M. Pawlik, MD, MPH, PhD ${ }^{5}$, Kenneth K. Tanabe, MD, FACS ${ }^{6}$, Mitchell C. Posner, MD $^{7}$, \\ Deborah Whippen, $\mathrm{BA}^{8}$, and Mark S. Roh, MD, MMM, FACS ${ }^{9}$
}

${ }^{1}$ Department of Surgical Oncology, University of Texas MD Anderson Cancer center, Houston, TX; ${ }^{2}$ Division of Surgical Oncology, Department of Surgery, University of Texas Southwestern Medical Center, Dallas, TX; ${ }^{3}$ Division of Breast Surgical Oncology, Department of Surgery and Pathology, University of Arkansas for Medical Sciences, Little Rock, AK; ${ }^{4}$ Department of Surgery, University of Louisville, Louisville, KY; ${ }^{5}$ Division of Surgical Oncology, Department of Surgery, The Johns Hopkins Hospital, Baltimore, MD; ${ }^{6}$ Division of Surgical Oncology, Massachusetts General Hospital Cancer Center and Harvard Medical School, Boston, MA; ${ }^{7}$ Department of Surgery, The University of Chicago, Chicago, IL; ${ }^{8}$ Editorial Office, Annals of Surgical Oncology, North Fort Myers, FL; ${ }^{9}$ UF Health Cancer Center, Orlando Health, Orlando, FL

The impact of a medical or scientific journal is often attributed to the 2-year citation index, as well as its rank order relative to other journals in its universe of subjects. These rankings are derived from impact factors based on a system devised by the Institute for Scientific Information (now Thomson Reuters) in the 1950s. An original purpose of the journal impact factor ranking was to provide an evaluative resource to medical libraries for decision making about which journals to include in their holdings. Currently, there is an active dialogue among scientific journals about the strengths and weaknesses of the impact factor ranking system as an evaluative tool. ${ }^{1-7}$ Indeed, some journal editors have been highly critical, even to the point of suggesting abandonment of the impact factor as a journal metric. ${ }^{4}$ Recently, the Editor of the Proceedings of The National Academy of Sciences stated that "the scientific community must not rely exclusively on the impact factor of journals." ${ }^{2}$ One shortcoming of a journal's impact factor is that it can be skewed by publication of an isolated, but highly cited paper. In addition, citation data do not distinguish between review articles (which usually

(C) Society of Surgical Oncology 2016

First Received: 24 April 2016;

Published Online: 22 June 2016

C. M. Balch, MD, FACS

e-mail: editor@asoeditorial.org generate a higher number of citations, including self-citations) versus original peer-reviewed scientific articles, or editorials (which are generally not counted as a source document in the denominator when calculating the impact factor, while the number of citations can be used in the numerator of the calculation of the mean citations per source article). The 2-year impact factor can also understate citations when a journal publishes high impact and highly cited articles late in the year; this is an issue of concern for the Annals of Surgical Oncology because important papers from the annual meeting of the Society of Surgical Oncology (SSO) and the American Society of Breast Surgeons are published late in the year. Because of the perceived limitations of the traditional journal ranking system, additional evaluative tools and schemas have been used to benchmark the value of medical journals. ${ }^{3,6,7}$

The utility of any given journal metric is dependent on the mission of the journal. The Annals of Surgical Oncology is a journal that serves to disseminate important research findings and practice-changing results in the field of oncology that are of interest to members of the SSO, as well as an educational value to the broad community of surgical oncology investigators, practitioners, and trainees worldwide.

In our view, the value of a medical journal such as the Annals of Surgical Oncology is best evaluated by a composite of benchmarks that better reflect its scientific and educational impact. Moreover, the traditional citation index 
TABLE 1 Characteristics of the Annals of Surgical Oncology for 2015

2718 manuscripts received
Most frequent source of submitted manuscripts (in rank order): US, Japan, China, Korea, and The Netherlands
1350 peer volunteers performed manuscript reviews in 2015
28 days average time to first decision
21 days average time from acceptance to electronic publication
$26 \%$ average acceptance rate
835 published articles in 13 issues and 3 supplements
1227 source items used for calculating the Thomson Reuters 2014 citation index
6084 published pages (print and electronic)
2-year (Thomson Reuters) impact factor: 3.930 (ranked 15 of 198 surgical titles)
5-year (Thomson Reuters) impact factor: 4.532 (ranked 11 of 198 surgical titles)
Google Scholar h5 index: 74 (ranked third of the top 20 surgery titles)
Eigenfactor ${ }^{\circledR}$ Score: 6.5 (ranked third of 154 surgical journals)
SCImago Journal Ranking: 1.81 (ranked 14 of 375 surgical titles)
2-year citations per document: 3.94
3-year citations per document: 4.10 (based on 2042 total documents)
Total 3-year citations: 7272 (ranked second of 375 surgical titles)
Full-text downloads for $2015: 562,751$
Overall visits by geographic region: Asia-Pacific, $34 \%$; North America, $31 \%$; Europe, $25 \%$; Latin America, $5 \%$

is not designed to measure the educational value of a journal, except as reflected in the citations by other authors. Rather, other benchmarks, such as full-text downloads, provide valuable information about readers that is not reflected in the citation index. For these reasons, we provide here the full spectrum of objective parameters on which the leadership of the SSO (which owns the journal) and the editorial leadership of Annals of Surgical Oncology critically evaluate the journal's value.

\section{ABOUT THE ANNALS OF SURGICAL ONCOLOGY}

Annals of Surgical Oncology has been in existence since January 1994, and has grown to be the largest and the most cited journal dedicated to surgical oncology topics. Its characteristics are listed in Table 1.

\section{2-YEAR AND 5-YEAR THOMSON REUTERS CITATION INDEX}

The citation index is a time-honored measurement of scientific value calculated based on peer-reviewed publications that cite articles published in the Annals of Surgical Oncology. The journal impact factor is calculated annually by Thomson Reuters in the InCites ${ }^{\mathrm{TM}}$ Journal Citation Reports $^{\circledR}$ (JCR; formerly by the Institute of Scientific Information). Despite the widespread acceptance of the citation index and its rankings of biomedical journals, it has some limitations or shortcomings regarding methodological
TABLE 2 Universe of top 20 ranking of the Thomson Reuters Citation Index among 'surgical journals'

General surgery audience (4)

Transplantation (4)

Surgical oncology (1)

Neurosurgery (1)

Orthopedics (1)

Cardiovascular/thoracic (2)

Vascular (1)

Plastic surgery (1)

Obesity (2)

Pathology (1)

Endoscopy (2)

aspects in the calculation of this index, including (i) lack of assessment of the quality or source of the citations; (ii) the inclusion of self-citations; (iii) the poor comparability between different scientific fields (e.g. including surgical pathology in the universe of surgical journals); and (iv) the analysis of mainly English-language publications. ${ }^{1}$ Using these methods, the number of citations of Annals of Surgical Oncology articles is compared to a diverse universe of 'surgical journals', which includes traditional journals with a general surgical audience, as well as those journals with a narrow surgical specialty audience, and even a surgical pathology audience (Table 2).

The 2-year journal impact factor is the average number of times that articles from the Annals of Surgical Oncology published in the past 2 years have been cited in the 
TABLE 3 Top 20 journal rankings for 2014 using the 5-year impact factor from the Journal Citation Reports database and published by Thomson Reuters

\begin{tabular}{ll}
\hline Journal & $\begin{array}{l}\text { 5-year impact } \\
\text { factor }\end{array}$ \\
\hline 1. Annals of Surgery & 8.844 \\
2. American Journal of Transplantation & 5.717 \\
3. Journal of Neurology and Neurosurgery & 5.550 \\
4. British Journal of Surgery & 5.522 \\
5. American Journal of Surgical Pathology & 5.328 \\
6. Journal of the American College of Surgeons & 5.263 \\
7. Journal of Heart and Lung Transplantation & 4.914 \\
8. JAMA Surgery & 4.893 \\
9. Endoscopy & 4.855 \\
10. Journal of Bone and Joint Surgery & 4.839 \\
11. Annals of Surgical Oncology & 4.532 \\
12. Annals of Thoracic Surgery & 4.104 \\
13. Journal of Thoracic and Cardiovascular Surgery & 4.068 \\
14. Surgery for Obesity and Related Diseases & 3.973 \\
15. JAMA Surgery & 3.936 \\
16. Liver Transplantation & 3.921 \\
17. Diseases of the Colon and Rectum & 3.685 \\
18. Transplantation & 3.604 \\
19. Journal of Neurosurgery & 3.573 \\
20. Obesity Surgery & 3.435 \\
\hline
\end{tabular}

Thomson Reuters JCR each year. The journal's impact factor is calculated by dividing the number of citations in the JCR year by the number of articles published in the two previous years. The 2014 2-year impact factor for the Annals of Surgical Oncology was 3.930, which ranks the journal 15th among 198 'surgical titles'.

The 5-year impact factor measures a more durable value of a published article because it measures how often a published source article is cited over the 5 years following publication by authors who submit articles to peer-reviewed journals. This index also mitigates the distortion of a 2-year citation index that does not account for publications that can take longer than 2 years to disseminate and respond to published works. Thus, the 5-year impact factor is calculated by dividing the number of citations in the Thomson Reuters JCR year by the total number of articles published in the 5 previous years. The 20155 -year impact factor for the Annals of Surgical Oncology was 4.532, which ranks the journal 11th among 198 'surgical titles' (Table 3).

\section{THE EIGENFACTOR ${ }^{\circledR}$ AND ARTICLE INFLUENCE SCORE}

The Eigenfactor (EF) Score and the Article Influence Score are calculated based on the citations received over a 5-year period. The Eigenfactor.org website provides a searchable database of EF scores and Article Influence Scores from over 8000 journals indexed in the Thomson Reuters Web of Science citation database, and covered in Thomson Reuters JCR since 1996. ${ }^{7-10}$

The EF Score reflects both the number of citations and also the prestige of the citation source. ${ }^{7-9}$ The citation data used at Eigenfactor.org come from Thomson Reuters JCR. Among the 154 surgical journals used to index and compare the EF Score, the Annals of Surgical Oncology ranks third, with an EF score of 6.5, after the Annals of Surgery (EF score of 8.4) and the Annals of Thoracic Surgery (EF score of 7.0) [Table 4].

The Article Influence Score measures the average influence, per article, of the papers in a journal..$^{7-10}$ As such, it is comparable to Thomson Reuters' widely used Impact Factor. However, in contrast to the Thomson Reuters JCR Impact Factor, where all citations are counted equally regardless of their source, each citation in the Article Influence Score is multiplied by the 'quality' of the citing journals, resulting in greater weights for citations that come from highly cited journals, and less weight to poorly cited journals. $^{7}$ Article Influence Scores are normalized so that the mean article in the entire Thomson Reuters $\mathrm{JCR}^{\circledR}$ database has an article influence of 1.00. The Article Influence Score for the Annals of Surgical Oncology is 1.4, which means that its articles have a $40 \%$ greater influence of the mean article in the Thomson Reuters JCR database.

\section{SCIMAGO JOURNAL RANK INDICATOR}

The SCImago Journal Rank Indicator (SJR) is based on the SCOPUS ${ }^{\circledR}$ database, which has much wider indexed journals compared with Thomson Reuters JCR. ${ }^{8-12}$ Compared with the Thomson Reuters JCR source database, the SCOPUS database includes a larger number of journals that originate from more countries, includes journals published in a greater variety of languages, calculates the 'prestige' of the citing journal (rather than the absolute number of citations), and does not include journal self-citations. ${ }^{11}$ The SJR is updated daily, uses a 3-year time window, and is an open access process that is available on the web at http:// www.scimagojr.com/journalsearch.php. In contrast, the JCR impact factor is only published once a year. In addition, some high-quality journals that are included in the broader SCOPUS database are not indexed by Thomson Reuters. Furthermore, SJR uses a denominator that includes all types of published items, whereas Thomson Reuters only considers original articles and review articles as citable items (or source items) from each journal.

According to the SJR index, the Annals of Surgical Oncology is ranked 14th among a larger universe of 375 
TABLE 4 The Eigenfactor score and Article Influence Score

\begin{tabular}{|c|c|c|}
\hline Journal & Eigenfactor & Article Influence Score \\
\hline Annals of Surgery & 8.4 & 3.2 \\
\hline Annals of Thoracic Surgery & 7.0 & 1.5 \\
\hline Annals of Surgical Oncology & 6.5 & 1.4 \\
\hline Journal of Thoracic and Cardiovascular Surgery & 6.1 & 1.6 \\
\hline American Journal of Transplantation & 5.9 & 2.0 \\
\hline Journal of Bone and Joint Surgery & 5.3 & 1.8 \\
\hline Neurosurgery & 4.4 & 1.1 \\
\hline Surgical Endoscopy & 4.3 & 0.9 \\
\hline Journal of Vascular Surgery & 4.3 & 1.0 \\
\hline Transplantation & 4.3 & 1.2 \\
\hline Clinical Orthopedics & 4.3 & 1.1 \\
\hline Journal of the American College of Surgeons & 4.1 & 2.0 \\
\hline Journal of Neurology and Neurosurgery & 3.9 & 2.0 \\
\hline British Journal of Surgery & 3.0 & 2.0 \\
\hline European Journal of Cardiothoracic Surgery & 3.7 & 1.1 \\
\hline Journal of Neurosurgery & 3.7 & 1.1 \\
\hline Plastic and Reconstructive Surgery & 3.6 & 0.9 \\
\hline American Journal of Surgical Pathology & 3.4 & 1.7 \\
\hline World Journal of Surgery & 3.0 & 0.9 \\
\hline Surgery & 2.9 & 1.3 \\
\hline
\end{tabular}

http://www.eigenfactor.org/projects/journalRank/

surgical journals worldwide. The SJR rankings of the top 20 surgical journals are listed in Table 5. Using the SJR data for the top 20 surgical journals, the Annals of Surgical Oncology ranked 4th in total documents published in 2014 (behind three cardiothoracic surgery journals) and second after the Annals of Thoracic Surgery in the total 'citable' publications over 3 years (Table 5). The 2-year average number of citations/citable documents published for the Annals of Surgical Oncology was 3.94 (exactly the same as the 2-year Thomson Reuters citation index), ranking it sixth behind the Annals of Surgery (8.2 citations/documents over 2 years), Journal of Neurology, Neurosurgery and Psychiatry (6.22), Journal of Heart and Lung Transplantation (5.58), American Journal of Surgical Pathology (5.57), British Journal of Surgery (5.43) and the Journal of the American College of Surgeons (4.8).

\section{TOTAL CITATIONS PER YEAR}

An important measure of a journal's scientific value is the total citations per year. While the 2- and 5-year impact factors determine the average number of citations per source article published, these factors do not take into account the total number of citations attributable to a specific journal, and thus favor journals that publish fewer manuscripts. Because the citation index uses an average (i.e. mean not median) value, its citation index may be derived from only a few published articles that may be highly cited, while the remainder of the articles are cited only occasionally.

For larger journals, the total citations more accurately reflect the scientific impact of large journals such as the $A n$ nals of Surgical Oncology, as demonstrated in Fig. 1 from the Thomson Reuters JCR database and Table 5 from the SCOPUS database. Compared with the Annals of Surgical Oncology (with 7272 total citations over 3 years), only the Annals of Surgery had more 'total citations' (7532 total citations over 3 years) using the SCOPUS database (Table 5). There are no comparable rankings of surgery journals for total citations from the Thomson Reuters JCR database.

\section{THE H5-INDEX (GOOGLE SCHOLAR)}

The h-index is an index that attempts to measure both the productivity and impact of a journal rather than individual articles. ${ }^{13}$ This tool uses Google Scholar as a data source instead of the Thomson Reuters Web of Knowledge. This metric is based on the articles published by a journal over the previous 5 calendar years with a minimum of 100 articles in this period. The ' $h$ ' represents the largest number of articles that have been cited ' $h$ ' times. For example, an h5 index of 5 would mean that the journal has published five articles that have been cited at least five times over a given 5-year period. As such, the h5 index cannot therefore 
TABLE 5 SJR rankings for 2014 of the top 20 surgical journals

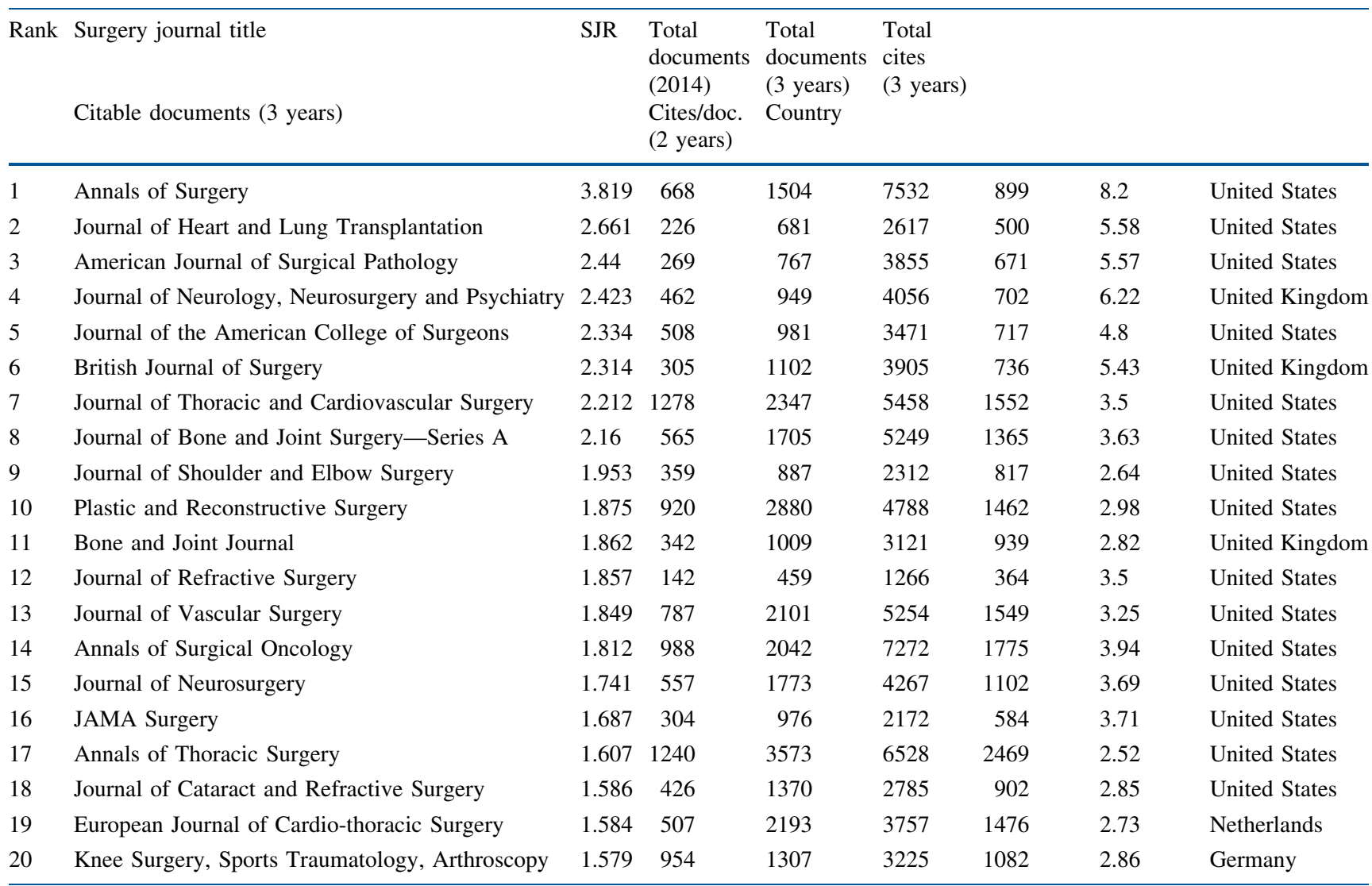

Source http://www.scimagojr.com, as of March 2016

SJR SCImago Journal Rank

be dominated by one or several highly cited articles. The h5 index provides a measure of sustained performance of an entire journal rather than individual articles published.

The h-index is intended to simultaneously measure the quantity (number of articles) and quality (impact or citations to those articles) of scientific impact. The h5 index for the Annals of Surgical Oncology for articles published from 2010 to 2014 was 74 (i.e. there were 74 articles published between 2010 and 2014 that have been cited at least 74 times). The Annals of Surgical Oncology is ranked number 3 among the universe of surgery journals (Table 6) and number 16 among oncology journals.

Both the h5 Google Scholar Index and the Eigenfactor ${ }^{\circledR}$ Index use different databases (i.e. Google vs. Thomson Reuters) but are similar in that they use a 5-year time horizon (which reflects more durable impact of journal articles), while only the EF Index takes into account the 'prestige' of the journals that are citing the article, and they eliminate self-citations. In both indexes, the Annals of Surgical Oncology ranks third among a somewhat different but overlapping universe of surgical journals (Tables 4, 6).

\section{MEASUREMENT OF A JOURNAL'S EDUCATIONAL IMPACT}

While the impact factor measures how often an author cites other published articles, it does not measure how often readers may view a published article for educational purposes while not citing the article in a publication. This situation likely occurs commonly for publications such as invited editorials and educational articles that are often downloaded but not necessarily cited. Measuring educational impact with full-text downloads is also relevant for journals with a large global audience, including those readers from countries that may not publish a robust number of peer-reviewed articles compared with readers from other countries.

The Annals of Surgical Oncology is now available electronically through 9000 institutions throughout the world through the consortia agreements by its publisher, Springer. Over the past 10 years, the number of full-text downloads has increased significantly to over 500,000 articles a year (Fig. 2). The value of electronic distribution 
TABLE 6 The h5 ranking of surgical journals in 2015 by Google Scholar ${ }^{\mathrm{a}}$ and the Eigenfactor/Article Influence Score for $2014^{\mathrm{b}}$

\begin{tabular}{|c|c|c|c|}
\hline Journal & h5-index & Eigenfactor & Article Influence Score \\
\hline 1. Annals of Surgery & 91 & 8.4 & 3.2 \\
\hline 2. Journal of Vascular Surgery & 80 & 4.3 & 1.0 \\
\hline 3. Annals of Surgical Oncology & 74 & 6.5 & 1.4 \\
\hline 4. Surgical Endoscopy & 73 & 4.3 & 0.9 \\
\hline 5. British Journal of Surgery & 70 & 3.9 & 2.0 \\
\hline 6. Journal of the American College of Surgeons & 67 & 4.1 & 2.0 \\
\hline 7. JAMA Surgery (Archives of Surgery) & 57 & 2.1 & 1.9 \\
\hline 8. Journal Trauma and Acute Care Surgery & 56 & 1.5 & 0.8 \\
\hline 9. Obesity Surgery & 55 & 1.0 & 0.8 \\
\hline 10. World Journal of Surgery & 55 & 3.0 & 0.9 \\
\hline 11. Surgery & 51 & 2.9 & 1.3 \\
\hline 12. Journal of Gastrointestinal Surgery & 49 & 2.4 & 0.9 \\
\hline 13. European Journal of Vascular and Endovascular Surgery & 47 & 1.9 & 1.1 \\
\hline 14. American Journal of Surgery & 47 & 2.3 & 1.0 \\
\hline 15. Surgery for Obesity and Related Diseases & 46 & 1.1 & 1.1 \\
\hline 16. Journal of Surgical Research & 46 & 2.7 & 0.6 \\
\hline 17. Journal of Surgical Oncology & 45 & 2.0 & 0.9 \\
\hline 18. European Journal of Surgical Oncology & 44 & - & - \\
\hline 19. Journal of Endourology & 42 & 1.2 & 0.5 \\
\hline 20. Journal of Hepato-Biliary-Pancreatic Sciences & 40 & 0.7 & 0.7 \\
\hline
\end{tabular}

${ }^{a}$ https://scholar.google.com/citations?view_op=top_venues\&hl=en\&vq=med_surgery

${ }^{\mathrm{b}}$ http://www.eigenfactor.org/projects/journalRank

FIG. 1 Number of citations, source items, and 5-year impact factor by year

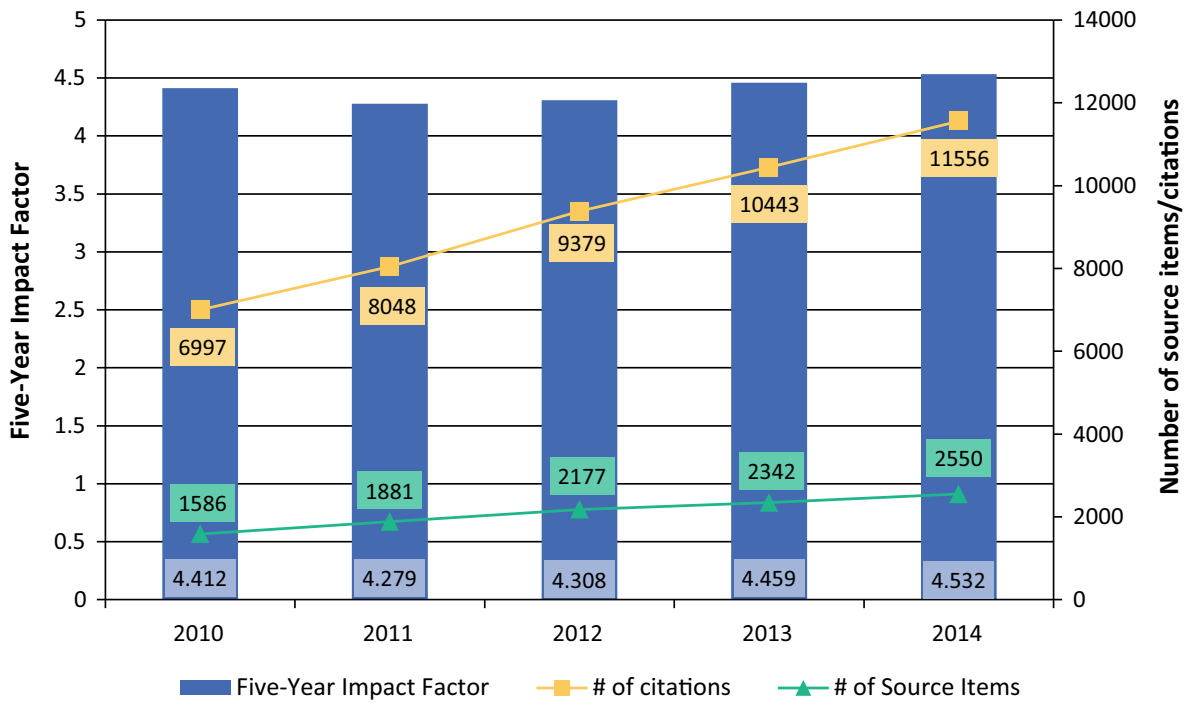

of the journal (compared with print distribution) is evident in the 562,751 full-text downloads in 2015 alone. With an average of 7.5 pages per article, the total downloads translate into over 4,200,000 printed pages of content. In contrast to the electronic distribution of the journal, the print version of the Annals of Surgical Oncology has a circulation of approximately 5000 subscribers, distributed largely in North America and sparsely in a few other countries in Europe and Asia. For this reason, we use fulltext downloads and page views as a measure of the educational value of the Annals of Surgical Oncology (Fig. 2). 


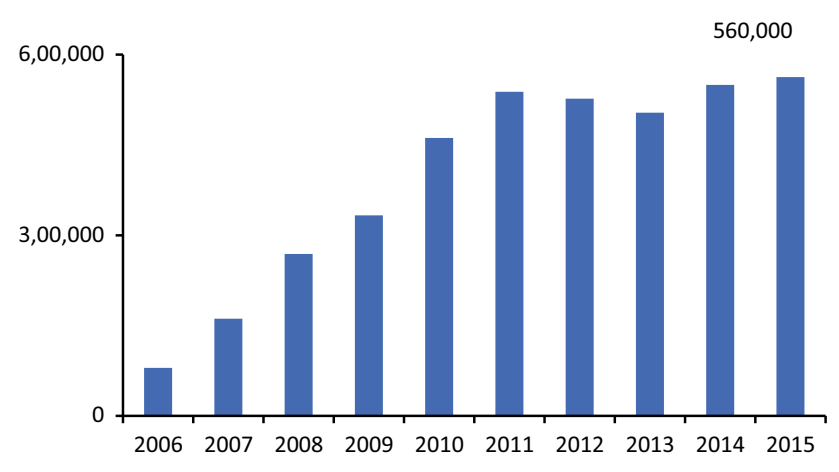

FIG. 2 Annals of Surgical Oncology usage over the past 10 years

\section{ALTMETRICS SCORE AS A MEASURE OF 'SOCIAL IMPACT'}

Altmetrics or 'alternative metrics' are offering new evaluation alternatives. One of these is a journal's reputation made via their footprint on the social web, including article mentions on Twitter, Facebook, Google+, Reddit, Blogs, news outlets, and Faculty of 1000 Reviews. In 2015, the Annals of Surgical Oncology had 1597 'article mentions' according to data provided by Altmetrics. The Altmetrics data provide additional research-impact indices that will be used more in the future as the data matures. The Annals of Surgical Oncology now uses a Twitter account @ AnnSurgOncol to rapidly disseminate articles as they are published online.

\section{CONCLUSION}

While the Annals of Surgical Oncology and other journals are measured and compared for their scientific value with the citation index, this benchmark does not necessarily reflect the entire 'impact' or educational value of a journal's publications. The composite of 2- and 5-year impact factors, the total number of citations per year, and the $\mathrm{h} 5$ index are important metrics that reflect the scientific value of the journal, as evidenced by the citation of Annals of Surgical Oncology publications by authors of peer-reviewed articles. In addition, full-text downloads are measured and tracked as benchmarks of the educational value of this publication. Use of composite measures to benchmark the impact and educational value of published articles will become increasingly important as the global audience of readers increases and the importance of social media in scientific publishing continues to expand.

Based on the composite of journal rankings, especially those that measure the more durable impact of articles cited over a longer term horizon of 3-5 years, and based on the total number of citations, the Annals of Surgical Oncology is the top journal among a large universe of surgical journals published throughout the world. The Annals of Surgical Oncology also serves an important educational benefit globally, as measured by the robust number of fulltext downloads.

We encourage other medical and scientific journals to use benchmarks beyond the 2-year citation index that is commonly used as a sole reflection of a medical journal's impact. The worth and value of our journals are more multidimensional than this single index of benchmarking used to measure a medical journal's value to its readers.

ACKNOWLEDGMENT The editorial leadership of the Annals of Surgical Oncology thank Sean Beppler, Yvonne Chan, and Antoinette Cimino at Springer for their input and review of this editorial.

\section{REFERENCES}

1. Seglen PO. Why the impact factor of journals should not be used for evaluating research. BMJ. 1997;314:498-502.

2. Verma IM. Impact, not impact factor. Proc Natl Acad Sci USA. 2015; 112:7875-7876.

3. The impact factor game. It is time to find a better way to assess the scientific literature. PLOS Med. 2006;3:e291.

4. Coats AJ, Shewan LG. Impact factor: vagaries, inconsistencies and illogicalities; should it be abandoned? Nucl Med Rev Cent East Eur. 2012;15:132-6.

5. Smith R. Commentary: the power of the unrelenting impact factor: is it a force for good or harm? Int J Epidemiol. 2006;35:1129-30.

6. Brown T. Journal quality metrics: options to consider other than impact factors. Am J Occup Ther. 2011;65:346-50.

7. Rizkallah J, Sin DD. Integrative approach to quality assessment of medical journals using impact factor, eigenfactor, and article influence scores. PLoS One. 2010;5:e10204.

8. Cantin M, Munoz M, Roa I. Comparison between impact factor, Eigenfactor score, and SCImago journal rank indicator in anatomy and morphology journals. Int J Morph. 2015;33:1183-8.

9. Kianifar H, Sadeghi R, Zarifmahmoudi L. Comparison between impact factor, Eigenfactor metrics, and SCimago journal rank indicator of pediatric neurology journals. Acta Inform Med. 2014;22:103-6.

10. Ramin S, Sarraf Shirazi A. Comparison between impact factor, SCImago journal rank indicator and Eigenfactor score of nuclear medicine journals. Int J Cardiol. 2015;201:454-6.

11. Falagas ME, Kouranos VD, Arencibia-Jorge R, et al. Comparison of SCImago journal rank indicator with journal impact factor. FASEB J. 2008;22:2623-8.

12. Jamali J, Salehi-Marzijarani M, Ayatollahi SM. factors affecting journal quality indicator in Scopus (SCImago Journal Rank) in obstetrics and gynecology journals: a longitudinal study (19992013). Acta Inform Med. 2014;22:385-8.

13. Kulasegarah J, Fenton JE. Comparison of the $h$ index with standard bibliometric indicators to rank influential otolaryngologists in Europe and North America. Eur Arch Otorhinolaryngol. 2010;267:455-8. 